PROCEEDINGS OF THE

AMERICAN MATHEMATICAL SOCIETY

Volume 139, Number 11, November 2011, Pages 4017-4026

S 0002-9939(2011)10820-7

Article electronically published on March 28, 2011

\title{
ON SOME CONSTANTS FOR OSCILLATION AND STABILITY OF DELAY EQUATIONS
}

\author{
LEONID BEREZANSKY AND ELENA BRAVERMAN
}

(Communicated by Yingfei Yi)

\begin{abstract}
We discuss the famous constants of $1 / e, 1,3 / 2$ in necessary and/or sufficient oscillation and stability conditions for delay differential equations with one or more delays:

$$
x^{\prime}(t)=-\sum_{k=1}^{m} a_{k}(t) x\left(t-h_{k}(t)\right)
$$

including equations with oscillatory coefficients. Some counterexamples (which refer to necessary oscillation and stability conditions) are presented and open problems are stated.
\end{abstract}

\section{INTRODUCTION}

It is well known that the differential equation

$$
x^{\prime}(t)=-a x(t-h), a>0,
$$

is uniformly stable if $a h \leq \pi / 2$ and is exponentially stable if $a h<\pi / 2$. This is not valid anymore for the equation with a variable delay and a variable coefficient,

$$
x^{\prime}(t)=-a(t) x(t-h(t)),
$$

where $a(t)$ and $h(t)$ are nonnegative bounded continuous functions, $0 \leq a(t) \leq \alpha$, $0 \leq h(t) \leq q$. If $\alpha q \leq 3 / 2$, then equation (1.1) is uniformly stable. If $0<\beta \leq$ $a(t) \leq \alpha$ and $\alpha q<3 / 2$, then it is exponentially stable; see 1, 2, 3.

The above conditions can be generalized [4:

the inequality $\sup _{t \geq 0} \int_{t}^{t+q} a(s) d s \leq 3 / 2$ implies uniform stability, while the conditions

$$
\sup _{t \geq 0} \int_{t}^{t+q} a(s) d s<3 / 2, \liminf _{t \geq 0} \int_{t}^{t+q} a(s) d s>0,
$$

imply exponential stability.

Let us note that constants $\pi / 2$ and $3 / 2$ are sharp.

Received by the editors December 17, 2009 and, in revised form, September 30, 2010.

2010 Mathematics Subject Classification. Primary 34K11, 34K20.

Key words and phrases. Delay equations, stability, oscillation, non-oscillation, oscillatory coefficients.

The first author was partially supported by the Israeli Ministry of Absorption.

The second author was partially supported by an NSERC Research Grant and is the corresponding author.

(C)2011 American Mathematical Society Reverts to public domain 28 years from publication 
Consider the equation with several variable delays and constant coefficients:

$$
x^{\prime}(t)=-\sum_{j=1}^{m} a_{j} x\left(t-h_{j}(t)\right),
$$

where $a_{j}>0,0 \leq h_{j}(t) \leq q_{j}$. Then inequalities $\sum_{j=1}^{m} a_{j} q_{j} \leq \frac{3}{2}$ and $\sum_{j=1}^{m} a_{j} q_{j}<\frac{3}{2}$ are sufficient for uniform and exponential stability, respectively [5]. If all $h_{j}(t)$ are constant, then $3 / 2$ can be replaced by $\pi / 2$ [6].

However, these results are not valid if coefficients are not constant. Consider the equation

$$
x^{\prime}(t)=-\sum_{j=1}^{m} a_{j}(t) x\left(t-h_{j}(t)\right),
$$

where $0 \leq a_{j}(t)<\alpha_{j}, 0 \leq h_{j}(t) \leq q_{j}$. Then

$$
\sum_{j=1}^{m} \alpha_{j} q_{j} \leq 1
$$

is sufficient for uniform stability, and constant 1 is sharp, as demonstrated in [5].

Let us remark that $(3 / 2)$-constant is still relevant if we consider the "worst" delay only: if

$$
\max _{k} q_{k} \sum_{j=1}^{m} \alpha_{j} \leq \frac{3}{2}
$$

then the equation is uniformly stable and the inequalities

$$
\max _{k} q_{k} \sum_{j=1}^{m} \alpha_{j}<\frac{3}{2}, \liminf _{t \rightarrow \infty} \sum_{j=1}^{m} a_{j}(t)>0
$$

imply exponential stability.

We would like to answer the following question: does there exist a number $A>0$ such that the inequality

$$
\limsup _{t \rightarrow \infty} \int_{t-h(t)}^{t} a(s) d s \geq A
$$

implies instability of equation (1.1) with one variable delay and a positive coefficient?

Another objective of the present paper is to discuss constants which lead to either oscillation or non-oscillation. For delay differential equations the following result is well known [7:

If

$$
\limsup _{t \rightarrow \infty} \int_{t-\max _{k} h_{k}(t)}^{t} \sum_{j=1}^{m} a_{j}^{+}(s) d s<\frac{1}{e},
$$

then there exists an eventually positive solution of the equation

$$
\dot{x}(t)+\sum_{k=1}^{m} a_{k}(t) x\left(t-h_{k}(t)\right)=0 .
$$


Here $1 / e$ is the best possible constant since the equation

$$
\dot{x}(t)+x(t-\tau)=0
$$

is oscillatory for $\tau>1 / e$.

In the monograph 7 for the equation

$$
\dot{x}(t)+a(t) x(t-\tau)=0, a(t) \geq 0, \tau>0,
$$

the authors constructed a counterexample which shows that condition (1.3) is not necessary for non-oscillation of equation (1.4).

By Theorem 3.4.3 in 7 the inequality

$$
\limsup _{t \rightarrow \infty} \int_{t-\min _{k} h_{k}(t)}^{t} \sum_{j=1}^{m} a_{j}(s) d s<1
$$

is necessary for non-oscillation of equation (1.4), where $a_{j}(t) \geq 0$.

In the present paper we study non-oscillation when the above limit is between $1 / e$ and 1 and we also discuss constants arising in stability theory.

The paper is organized as follows. Section 2 deals with non-oscillation constants. It contains a new sufficient non-oscillation condition when the limit in (1.6) is between $1 / e$ and 1 . We demonstrate that even for a constant delay any limit between $1 / e$ and 1 can be attained for a non-oscillatory equation. Section 3 presents a justification of the fact that any $A \in(0,2)$ can be chosen in (1.2) such that exponential stability of (1.1) is still possible. Further, we prove that no $A$ can be chosen (even for equations with a constant coefficient) such that (1.2) implies instability. Finally, in Section 4 we consider equation (1.1) with an oscillating coefficient $a(t)$.

\section{Oscillation: From $\frac{1}{e}$ TO 1}

Let us present sufficient non-oscillation conditions for equation (1.4) when the number

$$
\limsup _{t \rightarrow \infty} \int_{t-\min _{k} h_{k}(t)}^{t} \sum_{j=1}^{m} a_{j}(s) d s
$$

is between $1 / e$ and 1 .

We consider a scalar delay differential equation (1.4) under the following conditions:

(a1) $a_{k}, k=1, \cdots, m$, are Lebesgue measurable locally essentially bounded functions;

(a2) $h_{k}:[0, \infty) \rightarrow R$ are Lebesgue measurable functions, $h_{k}(t) \geq 0$,

$$
\limsup _{t \rightarrow \infty}\left[t-h_{k}(t)\right]=\infty, \quad k=1, \cdots, m .
$$

Further, we will apply the following non-oscillation condition.

Lemma 2.1 (7]). If there exists an eventually non-negative locally integrable solution of the inequality

$$
u(t) \geq \sum_{k=1}^{m} a_{k}^{+}(t) \exp \left\{\int_{t-h_{k}(t)}^{t} u(s) d s\right\},
$$

where $a^{+}=\max \{a, 0\}$, then equation (1.4) is non-oscillatory. 
Consider equation (1.4) with constant delays:

$$
\dot{x}(t)+\sum_{k=1}^{m} a_{k}(t) x\left(t-\tau_{k}\right)=0, \tau_{k}>0 .
$$

Theorem 2.2. Suppose that there exists $n_{0} \geq 0$ and a sequence $\left\{\lambda_{j}\right\}_{j=n_{0}-1}^{\infty}$ of positive numbers such that

$$
\sum_{k=1}^{m} a_{k}^{+}(t) \leq \lambda_{n} e^{-\left[\lambda_{n-1}(n \tau-t)+\lambda_{n}(t-(n-1) \tau)\right]},(n-1) \tau<t \leq n \tau, n \geq n_{0},
$$

where $\tau=\max _{k} \tau_{k}$. Then equation (2.2) is non-oscillatory.

Proof. Let us demonstrate that the function

$$
u(t)=\lambda_{n},(n-1) \tau<t \leq n \tau, n \geq n_{0},
$$

is a solution of inequality (2.1) for $t \geq n_{0} \tau$.

In the interval $(n-1) \tau \leq t \leq n \tau$ we have

$$
\begin{aligned}
& \exp \left\{\int_{t-\tau_{k}}^{t} u(s) d s\right\} \leq \exp \left\{\int_{t-\tau}^{t} u(s) d s\right\} \\
& =\exp \left\{\int_{t-\tau}^{(n-1) \tau} \lambda_{n-1} d s+\int_{(n-1) \tau}^{t} \lambda_{n} d s\right\} \\
& =\exp \left\{\left(\lambda_{n-1}(n \tau-t)\right)+\lambda_{n}(t-(n-1) \tau)\right\} .
\end{aligned}
$$

Hence inequality (2.1) is equivalent to (2.3), which completes the proof.

Remark 2.3. By applying comparison theorems [7, Theorem 2.2 can be extended to equations with variable delays $0 \leq h_{k}(t) \leq \tau_{k}$.

Example 2.4. Consider equation (1.5), where $\tau=1$,

$$
a(t)= \begin{cases}e^{-(2 n-t+1)}, & 2 n-1 \leq t<2 n, n \geq 1, \\ 2 e^{-(t-2 n+1)}, & 2 n \leq t<2 n+1, n \geq 0 .\end{cases}
$$

If $\lambda_{2 n}=1, \lambda_{2 n-1}=2$, then all conditions of Theorem 2.2 hold; hence equation (1.5) is non-oscillatory. Since

$$
\limsup _{t \rightarrow \infty} \int_{t-1}^{t} a(s) d s=2\left(e^{-1}-e^{-2}\right)>e^{-1},
$$

then condition (1.3) does not hold for equation (1.5) and thus is not necessary for non-oscillation of this equation.

We apply the idea of Example 2.4 to prove the following result.

Theorem 2.5. For any $\alpha \in(1 / e, 1)$ there exists a non-oscillatory equation (1.5) with $a(t) \geq 0$ such that

$$
\limsup _{t \rightarrow \infty} \int_{t-\tau}^{t} a(s) d s=\alpha
$$


Proof. It is sufficient to prove the statement of the theorem for $\tau=1$. Let $\lambda>0$, $a>1$, and consider equation (1.5) with $\tau=1$,

$$
a(t)= \begin{cases}\lambda \exp \{-[2 \lambda(a-1) n-\lambda(a-1) t+\lambda]\}, & 2 n-1 \leq t<2 n, n \geq 1, \\ \lambda a \exp \{-[\lambda(a-1) t-2 \lambda(a-1) n+\lambda]\}, & 2 n \leq t<2 n+1, n \geq 0 .\end{cases}
$$

If $\lambda_{2 n}=\lambda, \lambda_{2 n-1}=\lambda a$, then all conditions of Theorem 2.2 hold; hence equation (1.5) is non-oscillatory. We have

$$
\limsup _{t \rightarrow \infty} \int_{t-1}^{t} a(s) d s \geq \int_{2 n}^{2 n+1} a(s) d s=\frac{a}{a-1}\left(e^{-\lambda}-e^{-\lambda a}\right)
$$

and the maximum of the function $f(\lambda)=\frac{a}{a-1}\left(e^{-\lambda}-e^{-\lambda a}\right)$ is attained at $\lambda_{0}=\frac{\ln a}{a-1}$ and equals $\max f(\lambda)=f\left(\lambda_{0}\right)=e^{-\lambda_{0}}$. Since

$$
\lim _{a \rightarrow 1} \frac{\ln a}{a-1}=1, \lim _{a \rightarrow \infty} \frac{\ln a}{a-1}=0,
$$

then assuming $\lambda=\frac{\ln a}{a-1}$ in the definition of function $a(t)$, we obtain

$$
\limsup _{t \rightarrow \infty} \int_{t-1}^{t} a(s) d s=\exp \left\{-\frac{\ln a}{a-1}\right\},
$$

where

$$
\limsup _{a \rightarrow 1} \limsup _{t \rightarrow \infty}^{t} a(s) d s=\frac{1}{e}, \lim _{a \rightarrow \infty} \limsup _{t \rightarrow \infty} \int_{t-1}^{t} a(s) d s=1 .
$$

Evidently $\limsup _{t \rightarrow \infty} \int_{t-1}^{t} a(s) d s$ is a continuous function of $a$; thus it takes all values in the interval $(1 / e, 1)$.

Let us note that if in (1.6) we substitute the minimal delay by the maximal delay,

$$
\limsup _{t \rightarrow \infty} \int_{t-\max _{k} h_{k}(t)}^{t} \sum_{j=1}^{m} a_{j}(s) d s<1,
$$

this condition is not necessary for non-oscillation.

Example 2.6. The equation

$$
x^{\prime}(t)+0.01 x(t-10)+0.3 x(t)=0
$$

is non-oscillatory, since the characteristic equation $\lambda+0.01 e^{-10 \lambda}+0.3=0$ has two real roots, $\lambda_{1} \approx-0.3261$ and $\lambda_{2} \approx-0.5536$. However, (2.6) is not satisfied since $10(0.01+0.3)=3.01>1$.

\section{Stability: are $\frac{3}{2}$ And $\frac{\pi}{2}$ Strict instability Limits?}

First of all, we present the following result which allows us to expand the set of constants in (1.6) such that equation (1.1) may be stable up to the limit of 2 .

Lemma 3.1 ([8]). Suppose $a(t) \geq 0$,

$$
\begin{gathered}
\liminf _{t \rightarrow \infty} a(t)>0, \\
\limsup _{t \rightarrow \infty} h(t)<\infty,
\end{gathered}
$$


and there exists $r(t) \geq 0$ such that the equation

$$
\dot{x}(t)+a(t) x(t-r(t))=0
$$

is non-oscillatory. If

$$
\limsup _{t \rightarrow \infty}\left|\int_{t-h(t)}^{t-r(t)} a(s) d s\right|<1,
$$

then equation (1.1) is exponentially stable.

By choosing an appropriate $r(t)=\tau$ and the same coefficient as in the proof of Theorem 2.5, we can for each $\alpha<1$ construct a non-oscillatory equation (3.3) such that

$$
\limsup _{t \rightarrow \infty} \int_{t-\tau}^{t} a(s) d s=\alpha .
$$

Furthermore, applying Lemma 3.1, for any $\beta<2$ we can construct an exponentially stable equation with

$$
\limsup _{t \rightarrow \infty} \int_{t-\tau}^{t} a(s) d s=\beta
$$

Example 3.2. Consider equation (1.5), where $a(t)$ is defined in (2.5), and

$$
a=e^{3}, \lambda=\frac{\ln a}{a-1} \approx 0.157187 .
$$

Then (1.5) has a non-oscillatory solution and

$$
\limsup _{t \rightarrow \infty} \int_{t-1}^{t} a(s) d s=\int_{2 n}^{2 n+1} a(s) d s=\exp \left\{-\frac{\ln a}{a-1}\right\} \approx 0.854544, n=0,1, \cdots .
$$

Since

$$
\int_{2 n-1}^{2 n} a(s) d s=\frac{1}{a}\left[e^{-\lambda}-e^{-\lambda a}\right] \approx 0.042545
$$

and $a(t)$ is 2-periodic, then

$$
\int_{t-3}^{t-1} a(s) d s \approx 0.854544+0.042545<1 .
$$

By Lemma 3.1 the equation

$$
\dot{x}(t)+a(t) x(t-3)=0
$$

is exponentially stable while

$$
\limsup _{t \rightarrow \infty} \int_{t-3}^{t} a(s) d s=0.854544+0.042545+0.854544 \approx 1.751633>\frac{\pi}{2} .
$$

Is $A=2$ a limit? In other words, if $A>2$ in (1.2), does it imply instability? The following example demonstrates that the answer is negative.

Example 3.3. Consider the equation with a positive constant coefficient and a variable delay,

$$
\dot{x}(t)+a x(t-h(t))=0, t \geq 0, a>0,
$$


where $h(t)=\{t\}, t-h(t)=[t]$ (here $\{t\}$ and $[t]$ are the fractional and the integer parts of $t$, respectively) for $t \in(2 n, 2 n+1]$ and $h(t)=0$ for $t \in(2 n+1,2 n+2]$; i.e.

$$
\begin{aligned}
\dot{x}(t)+a x([t]) & =0, \quad t \in[2 n, 2 n+1), n=0,1,2, \cdots, \\
\dot{x}(t)+a x(t) & =0, \quad t \in[2 n+1,2 n+2), n=0,1,2, \cdots .
\end{aligned}
$$

Let $a>2$. Then

$$
x(1)=x(0)(1-a), x(2)=x(0)(1-a) e^{-a},|x(2)|<0.15|x(0)|,
$$

since for $f(a)=\left|(1-a) e^{-a}\right|=(a-2) e^{-a}$ we have

$$
\max _{a \in[2, \infty)} f(a)=\max _{a \in[2, \infty)}(a-1) e^{-a}=f(2)=e^{-2} \approx 0.1353
$$

and $|x(t)| \leq|x(0)(1-a)|$. Furthermore,

$$
|x(t)| \leq|x(0)(1-a)|\left(\frac{1}{e^{2}}\right)^{n}, t \in[2 n, 2 n+2] ;
$$

thus (3.5) is exponentially stable for any $a \geq 2$, while

$$
\limsup _{t \rightarrow \infty} \int_{t-h(t)}^{t} a(s) d s=\int_{t-1}^{t} a d s=a
$$

can be any positive number, which demonstrates that there is no upper bound for the integral such that above this limit all equations will be unstable.

\section{EQUations With an OSCillatory COEFFicient AND OPEN PROBLEMS}

Finally, let us discuss stability and non-oscillation of (1.1) when the coefficient $a(t)$ can be oscillatory but in some sense its positive part "prevails".

Example 4.1. Consider (1.1) with

$$
a(t)=\left\{\begin{array}{ll}
\alpha+\beta, & t \in[2 n, 2 n+1), \\
-\alpha, & t \in[2 n+1,2 n+2),
\end{array} \quad h(t)= \begin{cases}\{t\}+1, & t \in[2 n, 2 n+1), \\
0, & t \in[2 n+1,2 n+2),\end{cases}\right.
$$

where $\alpha>0, \beta>0, n=0,1,2, \cdots$. Then equation (1.1) becomes

$$
x^{\prime}(t)= \begin{cases}-(\alpha+\beta) x(2 n-1), & t \in[2 n, 2 n+1), \\ \alpha x(t), & t \in[2 n+1,2 n+2) .\end{cases}
$$

Consider (4.1) for $t \geq 0$ with $x(s)=s+1$ for $s \leq 0$. Then $x(-1)=0, x(0)=1=$ $x(1)$,

$$
x(2)=e^{\alpha}, x(3)=e^{\alpha}-\alpha-\beta, \cdots, x(2 n+1)=x(2 n-1)\left[e^{\alpha}-\alpha-\beta\right]
$$

and

$$
\limsup _{t \rightarrow \infty} \int_{t-h(t)}^{t} a(s) d s=\int_{t-2}^{t} a(s) d s=\beta, \int_{0}^{\infty} a(s) d s=\infty .
$$

However, for any $\beta>0$ there exists $\alpha>0$ large enough such that $\left[e^{\alpha}-\alpha-\beta\right]>1$, which means that $x(2 n+1)>x(2 n-1)$, so the solution is unstable and nonoscillating. Thus there is no such constant $A$ that for equations with oscillating $a(t)$ the inequality

$$
\limsup _{t \rightarrow \infty} \int_{t-h(t)}^{t} a(s) d s<A
$$


implies stability, and this claim is still valid even in the case when two additional conditions hold:

$$
\liminf _{t \rightarrow \infty} \int_{t}^{t+H} a(s) d s>0 \text { for some } H>0, \quad \int_{0}^{\infty} a(s) d s=\infty .
$$

The first of the above conditions indicates that the positive part of $a(t)$ "prevails" over the negative part, and the second equality in the case of positive $a(t)$ guarantees that all non-oscillatory solutions converge to zero.

Some new sufficient stability results for (1.1) with oscillatory coefficients can be found in the recent paper [9].

In private discussions, Y. Domshlak and I. Stavroulakis proposed the following hypothesis.

Conjecture. Consider equation (1.1) with an oscillatory coefficient a $(t)$. The inequality

$$
\limsup _{t \rightarrow \infty} \int_{t-h(t)}^{t} a(s) d s<\frac{1}{e}
$$

implies non-oscillation of (1.1), and the condition

$$
\liminf _{t \rightarrow \infty} \int_{t-h(t)}^{t} a(s) d s>\frac{1}{e}
$$

implies oscillation of all solutions of equation (1.1).

The following example demonstrates that the second part is incorrect; moreover, there is no $A>0$ such that the inequality

$$
\liminf _{t \rightarrow \infty} \int_{t-h(t)}^{t} a(s) d s>A
$$

implies oscillation of all solutions. Thus the inequality (1.6) is no longer necessary for non-oscillation if the coefficient of (1.1) might become negative.

Example 4.2. Consider (1.1) with

$h(t)=\left\{\begin{array}{ll}2, & t \in[2 n, 2 n+1), \\ \{t\}+3, & t \in[2 n+1,2 n+2),\end{array} \quad a(t)= \begin{cases}-\alpha, & t \in[2 n, 2 n+1), \\ e^{\alpha}-1, & t \in[2 n+1,2 n+2),\end{cases}\right.$

where $\alpha>0, n=0,1,2, \cdots$. Then equation (1.1) becomes

$$
x^{\prime}(t)= \begin{cases}\alpha x(t-2), & t \in[2 n, 2 n+1), \\ -\left(e^{\alpha}-1\right) x(2 n-2), & t \in[2 n+1,2 n+2) .\end{cases}
$$

Thus, the solution of (4.5) with the initial function

$$
x(t)= \begin{cases}e^{\alpha(t+2)}, & t \in[-2,-1], \\ e^{\alpha}-\left(e^{\alpha}-1\right)(t+1), & t \in[-1,0],\end{cases}
$$

is two-periodic. Really,

$$
\begin{gathered}
x^{\prime}(t)=\alpha e^{\alpha t}, t \in[0,1], x(0)=1 \Rightarrow x(t)=e^{\alpha t}, t \in[0,1], \\
x^{\prime}(t)=-\left(e^{\alpha}-1\right) x(-2)=1-e^{\alpha}, t \in[1,2] \\
\Rightarrow x(t)=e^{\alpha}-\left(e^{\alpha}-1\right)(t-1), x(2)=1 .
\end{gathered}
$$


This solution is two-periodic and non-oscillatory, while

$$
\liminf _{t \rightarrow \infty} \int_{t-h(t)}^{t} a(s) d s=\int_{2 n-3}^{2 n} a(s) d s=e^{\alpha}-2 \alpha-1>\frac{1}{e}
$$

for $\alpha \geq \alpha_{0} \approx 1.4522$. Here $\alpha_{0}$ is the only positive solution of the equation $e^{x}-2 x-$ $1=1 / e$ (the function on the left-hand side is increasing). Moreover, lim inf exceeds $1 / e$ for $\alpha>1.4522$. Thus (4.5) has a non-oscillatory solution, while $A<e^{\alpha}-2 \alpha-1$ for some $\alpha$ in (4.4) can be any positive number.

Example 4.1 implies that for any $\alpha>0$ there exists a non-oscillatory equation (1.1) with oscillating $a(t)$ such that

$$
\limsup _{t \rightarrow \infty} \int_{t-h(t)}^{t} a(s) d s=\alpha .
$$

In particular, this means that the well-known oscillation condition for equations (1.1) with $a(t) \geq 0$,

$$
\limsup _{t \rightarrow \infty} \int_{t-h(t)}^{t} a(s) d s>1,
$$

is no longer valid for equations with oscillatory $a(t)$.

In the present paper we have demonstrated that some famous constants in the oscillation and stability theory do not work anymore if we consider instability or equations with oscillatory coefficients. Let us outline the problems which are still open.

(1) (Liz) Does there exist $A>0$ such that the inequality

$$
\liminf _{t \rightarrow \infty} \int_{t-h(t)}^{t} a(s) d s>A
$$

implies instability of equation (1.1) with $a(t) \geq 0$ ?

If the answer is positive, is $A=\pi / 2$ or a greater number?

(2) (Domshlak, Stavroulakis) Prove that the inequality

$$
\limsup _{t \rightarrow \infty} \int_{t-h(t)}^{t} a(s) d s \leq \frac{1}{e}
$$

implies non-oscillation of equation (1.1) with oscillating coefficient $a(t)$.

\section{ACKNOWLEDGEMENT}

The authors are grateful to Eduardo Liz for fruitful discussions.

\section{REFERENCES}

[1] A. D. Myshkis, On the solutions of linear homogeneous differential equations of the first order and stable type with retarded arguments (Russian), Matematicheskii Sbornik 28 (1951), 641-658. MR0043343(13:246c)

[2] J. A. Yorke, Asymptotic stability for functional differential equations, in "Seminar on Differential Equations and Dynamical Systems" (edited by G. S. Jones), 69-79, Lecture Notes in Mathematics, Springer-Verlag, 1968. MR0233050 (38:1373)

[3] J. C. Lillo, Oscillatory solutions of the equation $y^{\prime}(x)=m(x) y(x-n(x))$, J. Differential Equations 6 (1969), 1-36. MR0241780 (39:3119)

[4] T. Yoneyama, On the $3 / 2$ stability theorem for one dimensional delay-differential equations, J. Math. Anal. Appl. 125 (1987), 161-173. MR891356 (88e:34139)

[5] T. Krisztin, On stability properties for one-dimensional functional-differential equations, Funkcial. Ekvac. 34 (1991), 241-256. MR.1130462 (92k:34103) 
[6] T. Krisztin, Stability for functional-differential equations and some variational problems, Tohoku Math. J. 42 (1990), 407-417. MR.1066669 (91j:34125)

[7] I. Györi and G. Ladas, Oscillation Theory of Delay Differential Equations with Applications, The Clarendon Press, Oxford University Press, New York, 1991. MR 1168471 (93m:34109)

[8] L. Berezansky and E. Braverman, Explicit exponential stability conditions for linear differential equations with several delays, J. Math. Anal. Appl. 332 (2007), 246-264. MR2319658 (2008e:34175)

[9] L. Berezansky and E. Braverman, On exponential stability of a linear delay differential equation with an oscillating coefficient, Appl. Math. Lett. 22 (2009), 1833-1837. MR2558549 $(2010 \mathrm{j}: 34174)$

Department of Mathematics, Ben-Gurion University of the Negev, Beer-Sheva 84105, ISRAEL

Department of Mathematics and Statistics, University of Calgary, 2500 University Drive N.W., Calgary, AB T2N 1N4, Canada

E-mail address: maelena@math.ucalgary.ca 